\title{
THE UNIFICATION OF ASYMMETRY SIGNATURES OF TYPE Ia SUPERNOVAE
}

\author{
Justyn R. Maund ${ }^{1,2,8}$, Peter Höflich ${ }^{3}$, Ferdinando Patat ${ }^{4}$, J. Craig Wheeler ${ }^{5}$, Paula Zelaya $^{6}$, Dietrich Baade ${ }^{4}$, \\ Lifan Wang ${ }^{7}$, Alejandro Clocchiatti $^{6}$, and Jason Quinn ${ }^{6}$ \\ ${ }^{1}$ Dark Cosmology Centre, Niels Bohr Institute, University of Copenhagen, Juliane Maries Vej, DK-2100 Copenhagen Ø, Denmark; justyn@ dark-cosmology.dk \\ ${ }^{2}$ Department of Astronomy \& Astrophysics, University of California, Santa Cruz, CA 95064, USA \\ ${ }^{3}$ Department of Physics, Florida State University, Tallahassee, FL 32306-4350, USA; pah@ astro.physics.fsu.edu \\ ${ }^{4}$ ESO-European Organisation for Astronomical Research in the Southern Hemisphere, Karl-Schwarzschild-Str. 2, 85748 Garching b. München, Germany; \\ fpatat@eso.org; dbaade@eso.org \\ ${ }^{5}$ Department of Astronomy and McDonald Observatory, The University of Texas, 1 University Station C1402, Austin, TX 78712-0259, USA; \\ wheel@astro.as.utexas.edu \\ ${ }^{6}$ Departamento de Astronomía y Astrofísica, PUC Casilla 306, Santiago 22, Chile; aclocchi@ astro.puc.cl, pazelaya@astro.puc.cl, jquinn@ astro.puc.cl \\ ${ }^{7}$ Department of Physics, Texas A\&M University, College Station, TX 77843-4242, USA; wang@ physics.tamu.edu \\ Received 2010 July 30; accepted 2010 November 1; published 2010 December 1
}

\begin{abstract}
We present a compilation of the geometry measures acquired using optical and IR spectroscopy and optical spectropolarimetry to probe the explosion geometry of Type Ia supernovae (SNe Ia). Polarization measurements are sensitive to asymmetries in the plane of the sky, whereas line profiles in nebular phase spectra are expected to trace asymmetries perpendicular to the plane of the sky. The combination of these two measures can overcome their respective projection effects, completely probing the structures of these events. For nine normal $\mathrm{SNe}$ Ia, we find that the polarization of $\mathrm{Si}$ II $\lambda 6355$ at 5 days before maximum $\left(p_{\mathrm{SiII}}\right)$ is well correlated with its velocity evolution $\left(\dot{v}_{\mathrm{SiII}}\right)$, implying that $\dot{v}_{\mathrm{Si} \text { II }}$ is predominantly due to the asymmetry of the SNe. We find only a weak correlation between the polarization of Si II and the reported velocities $\left(v_{\text {neb }}\right)$ for peak emission of optical Fe II and Ni II lines in nebular spectra. Our sample is biased, with polarization measurements being only available for normal SNe that subsequently exhibited positive (i.e., redshifted) $v_{\text {neb }}$. In unison these indicators are consistent with an explosion in which the outer layers are dominated by a spherical oxygen layer, mixed with an asymmetric distribution of intermediate-mass elements. The combination of spectroscopic and spectropolarimetric indicators suggests a single geometric configuration for normal SNe Ia, with some of the diversity of observed properties arising from orientation effects.
\end{abstract}

Key words: supernovae: general - techniques: polarimetric - techniques: spectroscopic

Online-only material: color figures

\section{INTRODUCTION}

The importance of Type Ia supernovae (SNe Ia) as cosmological distance indicators has been demonstrated over the last 12 years (Riess et al. 1998; Perlmutter et al. 1999). Key to their utilization as probes of the universe's expansion is an understanding of their apparent homogeneity (with a magnitude dispersion at maximum light of $\sigma_{B} \sim 0.8$; Phillips 1993) and the role of the underlying explosion mechanism. Models to describe these events, as the explosion of a carbon-oxygen Chandrasekhar mass white dwarf in a single or double degenerate binary system (Branch et al. 1995), proceed with burning as deflagrations (Gamezo et al. 2004; Röpke et al. 2006), detonations, or a combination of the two: delayed detonations (Khokhlov 1991). These different mechanisms will imprint a signature on the resulting chemical abundances and geometries of the ejecta (Höflich et al. 2006).

The geometries of SNe Ia have been probed using different techniques. At early times, spectropolarimetry of these events has shown a wide array of asymmetries (see Wang \& Wheeler 2008 for a review), in the plane of the sky. In general, the continuum polarization, which probes the shape of the photosphere, is of order a few tenths of a percent, indicating departures from a spherical symmetry of $<10 \%$ (Höflich 1991; Wang et al. 1997). Significant line polarization is observed, showing the distribution of elements in the ejecta, predominantly for Ca II (e.g., for

\footnotetext{
8 Sophie and Tycho Brahe Fellow.
}

SN 2001el; Wang et al. 2003; Kasen et al. 2003) and the principal classification feature for SNe Ia, Si II $\lambda$ 6355. Wang et al. (2007) identified a correlation between the degree of polarization of Si II and the light curve decline rate parameter $\Delta m_{15}(B)$.

Nebular phase spectroscopy of SNe Ia in the optical and infrared (IR), when the ejecta are optically thin and the spectra are dominated by the Fe-group elements, can be used to reveal the structure at the center of the explosion; albeit projected onto the radial velocity direction (Höflich et al. 2006; Motohara et al. 2006; Gerardy et al. 2007; Maeda et al. 2010a). The almostunblended Fe line at $1.6 \mu \mathrm{m}$ shows peculiar line profiles such as flat topped profiles indicating material with central cavities, and Gerardy et al. (2007) observed identical profiles and features in mid-IR lines. This strongly supports that the line asymmetries are kinematic in nature and all but exclude explanations due to uncertainties in the atomic physics, which may lead to an underestimation of blends or optical thickness effects.

Maeda et al. (2010b) linked the asymmetries of the central layers with expansion velocities less than $\lesssim 3000 \mathrm{~km} \mathrm{~s}^{-1}$ with the outer layers. They found that the peak of the nebular emission line profiles, in the optical, exhibited blueshifts or redshifts, consistent with an asymmetric distribution along the line of sight. It was found that this kinematical offset was related to the evolution of the velocity of the absorption minimum of the Si II $\lambda 6355$ feature (parameterized by the temporal velocity gradient $\dot{v}_{\mathrm{Si} \text { II }}$ ) at early times; suggesting both are signatures of the departure of SNe Ia from simple spherical symmetry. 
Probing the inner structure and correlations with outer layers is central to answering key questions of supernova theory (for a review see Höflich 2006), such as whether the asymmetries in the inner and outer layers have a common physical origin. In this Letter, we discuss the correlations between geometry indicators derived from early and late spectroscopic observations and spectropolarimetric observations of $\mathrm{SNe} \mathrm{Ia}$, to approach a unified model of the behavior of these events.

\section{POLARIZATION AND THE VELOCITY GRADIENT OF Si II}

Wang et al. (2007) observed that the polarization of the Si II line for normal SNe Ia peaks at $\sim-5$ days (relative to $B$ light curve maximum), and this was further confirmed for SN 2006X (Patat et al. 2009). Values of $\dot{v}_{\mathrm{Si} \text { II }}$ for a range of SNe Ia were compiled from those reported by Benetti et al. (2005) and Maeda et al. (2010b). These were cross checked against the list of SNe Ia with the polarization of Si II $\lambda 6355$ reported at -5 days (Wang et al. 2007).

For the normal SN 2007le, the Si II polarization was estimated from spectropolarimetric observations at -9 and -4 days (P. Zelaya et al. 2011, in preparation), with $\dot{v}_{\mathrm{Si} \text { II }}=83 \pm$ $3 \mathrm{~km} \mathrm{~s}^{-1}$ day $^{-1}$ (Simon et al. 2009). The polarization of Si II $\lambda 6355$ for SNe 2002bf, $2003 \mathrm{hv}$, and $2009 \mathrm{dc}$, observed at $+3,+5$, and +5.6 days after $B$-maximum were acquired from Leonard et al. (2005), J. R. Maund et al. (2011, in preparation), and Tanaka et al. (2010), respectively (with values of $\dot{v}_{\mathrm{Si} \text { II }}$ given by Leonard et al. 2005, Leloudas et al. 2009, and Yamanaka et al. 2009). Given that the observations of these $\mathrm{SNe}$ were conducted later than -5 days, we assume that the reported polarizations represent lower limits of $p_{\mathrm{Si} \text { II }}$ at -5 days. For SN $2001 \mathrm{~V}$, we estimated $\dot{v}_{\mathrm{Si} \text { II }}=81 \pm 7 \mathrm{~km} \mathrm{~s}^{-1} \mathrm{day}^{-1}$ (using the data of Matheson et al. 2008), ${ }^{9}$ which is higher than the 20 $\mathrm{km} \mathrm{s}^{-1}$ day $^{-1}$ measured for the similar SN 1991T (Phillips et al. 1992; Taubenberger et al. 2008).

The Si II polarizations for the peculiarly faint 1991bg-like SNe 1999by and 2005ke were taken from Howell et al. (2001) and F. Patat et al. (2011, in preparation), respectively. The velocity evolution of $\mathrm{Si}$ II in this particular subclass has been found to be relatively homogeneous (Taubenberger et al. 2008). We derive an average value $\dot{v}_{\mathrm{Si} \text { II }}=105.8 \pm 8.6 \mathrm{~km} \mathrm{~s}^{-1} \mathrm{day}^{-1}$ from the reported velocities of four members of the subluminous subclass (SNe 1991bg, Turatto et al. 1996; 1999by, Vinkó et al. 2001; Howell et al. 2001; 2005bl, Taubenberger et al. 2008; and 2005ke, F. Patat et al. 2011, in preparation). In the absence of further spectroscopic data, we assume that this value of the deceleration of Si II is valid for SN 2005ke.

The relation between the velocity gradient of Si II and the associated polarization is shown in Figure 1. There is a distinct correlation for normal SNe Ia and, excluding SN 2004dt, we find $p_{\text {Si II }}=0.267+0.006 \times \dot{v}_{\text {Si II }}$ (with $\chi_{v}^{2}=1.07$, Pearson correlation coefficient $r=0.93, p=0.007$ for $N=9$ $\mathrm{SNe}$ being drawn from an uncorrelated random sample). The SN 1991T-like SN 2001V and the faint SNe 1999by and 2005ke are obvious outliers from this relation. In comparing $p_{\mathrm{Si} \text { II }}$ and the light curve decline rate parameter $\Delta m_{15}(B)$, Wang et al. (2007) found that $\mathrm{SNe} 1999$ by and $2005 \mathrm{ke}$ are significant outliers from the linear correlation found between these two parameters. SN $2004 \mathrm{dt}$ is not a significant outlier $(<3 \sigma)$, and including it in the

\footnotetext{
9 The data were acquired from the SUSPECT archive: http://suspect.nhn.ou.edu/ suspect/.
}

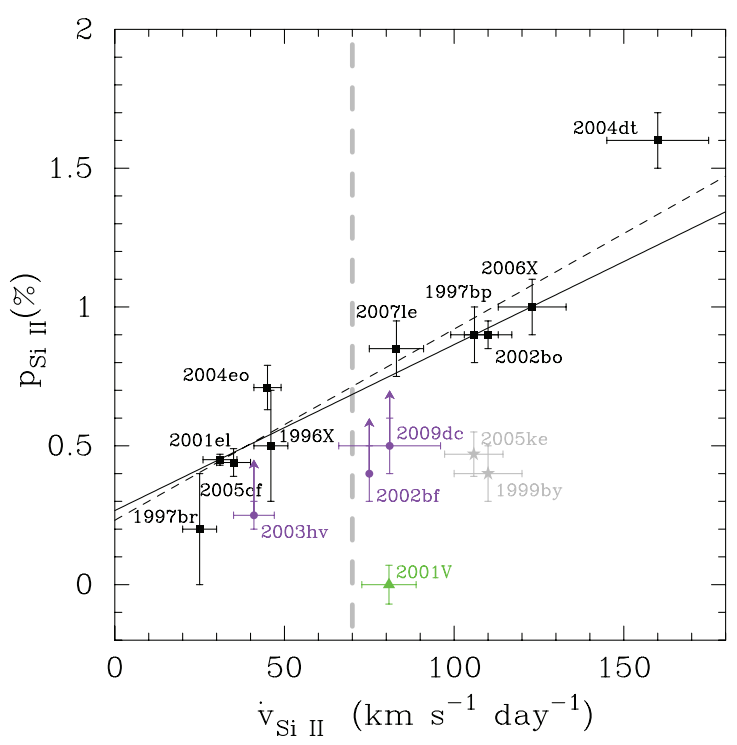

Figure 1. Observed line polarization of $\mathrm{Si}$ II $\lambda 6355$ at -5 days, relative to $B$ light curve maximum, as a function of the measured velocity gradient for the same line. The group of normal SNe Ia is indicated in black ( $\mathbf{\square})$, whereas the peculiarly faint SNe 1999 by and SN $2005 \mathrm{ke}$ are indicated in gray ( $\star$ ), and the single representative of the SN 1991T-like SNe 2001 V is indicated in green $(\boldsymbol{\Delta})$. Lower limits on the polarization of $\mathrm{Si}$ II for three $\mathrm{SNe}$ are presented in purple $(\bullet)$. The dashed gray line at $70 \mathrm{~km} \mathrm{~s}^{-1}$ day $^{-1}$ separates the HVG and LVG SNe Ia (Benetti et al. 2005). The solid black line indicates the best-fit straight line to the normal SNe Ia, while the dashed black line is for the best fit including SN 2004dt.

(A color version of this figure is available in the online journal.)

fit slightly changes the form of the relation. It may indicate that the relation at very high $\dot{v}_{\mathrm{Si} \text { II }}$ is no longer linear.

The dividing line between the high velocity gradient (HVG) and low velocity gradient (LVG) $\mathrm{SNe}$ is a deceleration of $\sim 70 \mathrm{~km} \mathrm{~s}^{-1}$ day $^{-1}$ in Figure 1 (see Benetti et al. 2005). The relative numbers in each group, however, are dictated by the selection effect of the number of SNe with both sufficiently dense early spectroscopy, to derive the Si II velocity evolution, and early spectropolarimetry. The gap between the HVG and LVG SNe may not, therefore, be a representative of significant differentiation between the two types of normal SNe Ia. The lower polarization limits provided by SNe 2002bf and 2009dc may fill this gap and indicate that SNe Ia form a continuous distribution on this diagram. Caution is required, however, as Leonard et al. (2005) suggest SN 2002bf may be related to the peculiar SN 2004dt, due to its high velocities, and Yamanaka et al. (2009) and Tanaka et al. (2010) suggest SN 2009dc may have arisen from a super-Chandrasekhar mass white dwarf progenitor.

\section{POLARIZATION AND THE NEBULAR PHASE VELOCITY OF Fe-GROUP ELEMENTS}

We examined the compilation of line-of-sight velocity offsets $v_{\text {neb }}$ for the peaks of the emission lines of [Fe II] $\lambda 7155$ and [Ni II] $\lambda 7378$, relative to their rest wavelength, provided by Maeda et al. (2010b). During the nebular phase ( $t>100$ days) these are presumed to be a measure of the expansion velocity of the central portions of the ejecta, representative of the products of the deflagration phase. For SN 2005df, we utilized the measurements of Gerardy et al. (2007) of the velocity offsets of [Ni III] 7.35 and $11.002 \mu \mathrm{m}$ and [Ni IV] $8.41 \mu \mathrm{m}$ at 118 days 


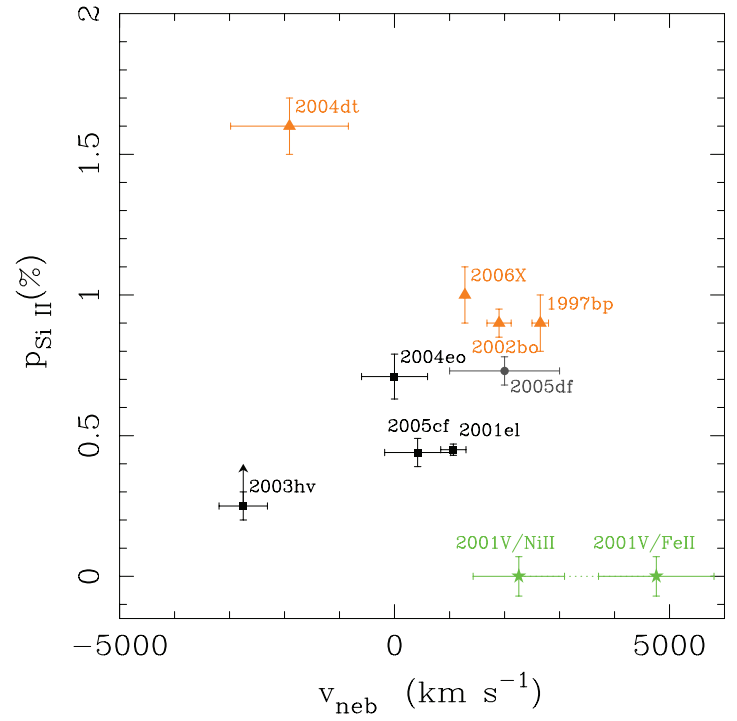

Figure 2. Observed line polarization of $\mathrm{Si}$ II $\lambda 6355$ at -5 days as a function of the nebular phase velocity offset. HVG SNe are indicated in orange $(\boldsymbol{\Lambda}), \mathrm{LVG}$ SNe are in black (ם), SN 2005df (without a measurement of $\dot{v}_{\mathrm{Si} \text { II }}$ ) is shown in gray $(\bullet)$, and the two measurements for SN $2001 \mathrm{~V}$ are shown in green $(\star)$.

(A color version of this figure is available in the online journal.)

(although Gerardy et al. do not observe an offset associated with [Co III] $11.89 \mu \mathrm{m}$ ).

The velocity offsets for SN $2001 \mathrm{~V}$ were determined for the optical Fe II and Ni II lines, from a spectrum acquired at 106 days (Matheson et al. 2008). We measured, however, different velocity offsets for the two species. This may indicate a more complicated differentiation of the distributions of Fe and $\mathrm{Ni}$ than observed for the normal HVG and LVG SNe Ia, and may be commensurate with SN $2001 \mathrm{~V}$ belonging to the subclass of 1991T-like SNe Ia.

In Figure 2, the polarization of Si II $\lambda 6355$ at early times is compared with the nebular velocity offset for events for which both data exist. This sample is limited by the small number of SNe Ia with both appropriate polarization measurements and late-time nebular spectra, with only one event exhibiting a blueshifted emission line profile (SN 2004dt). The remaining $\mathrm{SNe}$ Ia from our sample all have redshifted emission lines in their optical nebular spectra.

The apparent separation between the normal HVG and LVG SNe Ia in Figure 2 approximately reflects the correlation between $\dot{v}_{\mathrm{Si} \text { II }}$ and $p_{\mathrm{Si} \text { II }}$ established in Section 2, and the observation of Maeda et al. (2010b) that the nebular lines of SNe Ia with higher values of $\dot{v}_{\mathrm{Si} \text { II }}$ exhibit larger red displacements. The correlation between $v_{\text {neb }}$ and $p_{\mathrm{Si} \text { II }}$ for normal SNe Ia, excluding SN 2004dt and 2001V, is much weaker $(r=0.54, p=0.51$ for $N=7$ ) than the correlation of Figure 1. In this sample, based on measurements of $v_{\text {neb }}$ alone, it is difficult to distinguish between LVG and HVG normal SNe Ia (e.g., SNe 2001el and 2006X). Without further SNe with blueshifted nebular velocities and polarization measurements, it is difficult to ascertain the behavior in the blueshifted portion of the diagram. Given the relationship established in Section 2, those LVG SNe with negative $v_{\text {neb }}$ should appear in the lower left-hand quadrant of Figure 2, tending toward zero polarization.

Leloudas et al. (2009) measured a velocity gradient of $\dot{v}_{\text {Si II }}=41 \pm 6 \mathrm{~km} \mathrm{~s}^{-1}$ day $^{-1}$ for the normal SN 2003hv. This would suggest $p_{\mathrm{Si} \text { II }} \sim 0.5 \%$ at -5 days, which is consistent with adopting the observed polarization at +6 days (J. R. Maund et al. 2011, in preparation) of $0.25 \% \pm 0.05 \%$ as a lower limit. The implied decrease in the $p_{\mathrm{SiI}}$ between -5 and +6 days, however, is much slower than found for other normal SNe Ia (Wang et al. 2007). Motohara et al. (2006) and Leloudas et al. (2009) observed the [Fe II] line profiles for SN 2003hv as blueshifted ( $-2750 \pm 440 \mathrm{~km} \mathrm{~s}^{-1}$; Maeda et al. 2010b). Depending on the strength of $\mathrm{Si}$ II polarization at -5 days, if the polarization decreased dramatically, SN 2003hv may occupy a similar locus to that of SN 2004dt in Figure 2. This would support a trend relating $p_{\mathrm{Si} \text { II }}$ and $v_{\text {neb }}$ orthogonal to that apparent from the central group of points in Figure 2, with SN 2004dt and SN 2001V at the extremes of a general trend that runs through the cloud of normal HVG and LVG SNe Ia. If the polarization of SN 2003hv did not decrease substantially from -5 to +6 days, then SN 2003hv appears to conform with the trend identified for normal SNe Ia.

\section{DISCUSSION AND CONCLUSIONS}

Maeda et al. (2010b) observed a relationship between $\dot{v}_{\text {Si II }}$ and $v_{\text {neb }}$, and interpreted it as indicating a single asymmetric geometry for SNe Ia. This asymmetry gives rise to the apparent diversity among this $\mathrm{SN}$ class due to the different orientations at which individual $\mathrm{SNe}$ are observed. In considering polarimetric observations, we find a good linear correlation between $p_{\mathrm{SiII}}$, an established indicator of geometry, and $\dot{v}_{\mathrm{Si} \text { II }}$, implying that the latter is a signature of the geometry. The observed tight correlation between these two parameters for normal SNe Ia implies that the asymmetries probed by $p_{\mathrm{Si} \text { II }}$ are unlikely to be due to a line-forming region composed of small, randomly distributed clumps, rather it indicates the role of a general asymmetry of the ejecta. Similarly, in comparing $p_{\mathrm{Si} \text { II }}$ and $\Delta m_{15}(B)$, Wang et al. (2007) found their correlation suggested to be due to large-scale asymmetries.

We find that $p_{\mathrm{Si} \text { II }}$ shows a weak correlation with the later nebular velocity, that may indicate a possible correlation between the asymmetries inferred for the layers observed at early and late times. This correlation is expected to be weak, however, as $p_{\text {Si II }}$ and $v_{\text {neb }}$ probe orthogonal projections of the geometry, but may also reflect that the orientations of the principal asymmetries of late-time nebular features and early-time Si II are separated by $<180^{\circ}$. This suggests that in trying to understand the influences of geometry on measurable photometric and spectroscopic parameters, such as $\Delta m_{15}(B)$, it is preferable to use the early-time indicators $\dot{v}_{\mathrm{Si} \text { II }}$ and $p_{\mathrm{Si} \text { II }}$.

Benetti et al. (2005) suggested that the difference between HVG and LVG SNe Ia arises from the orientation at which the ejecta are viewed. They hypothesized that $\mathrm{SNe}$ Ia will be observed as HVG SNe if the ejecta approaching the observer were mixed with heavy elements, increasing the opacity, and keeping the photosphere at high velocities at early times (Höflich et al. 1993). In their model, LVG SNe Ia are observed from other angles, dominated by intermediate-mass elements (IMEs), through which the photosphere has already receded even at early times. A key consequence of this model is asymmetric excitation of the ejecta leading to an asymmetric photosphere, in particular for HVG SNe. This is in contrast to the low limits placed on the departure of the photospheres of SNe Ia from spherical symmetry from polarimetry of these events ( $p_{\text {cont }} \sim 0.2 \%-0.3 \%$; Wang et al. 2003, 1997; Patat et al. 2009).

It is significant that Si II, among other line features, is generally observed to be polarized in $\mathrm{SNe} \mathrm{Ia}$, whereas the continuum is not. This requires that the line-forming region 
be asymmetrically distributed across a spherical photosphere (Kasen et al. 2003; Leonard et al. 2005; Maund et al. 2010).

Benetti et al. (2005) and Maeda et al. (2010b) interpret the velocity of Si II $\lambda 6355$ as the photospheric velocity, which depends on the degree of mixing of Fe-group elements and the opacity in the outer layers of the ejecta. Patat et al. (1996) find, however, that the S II "W" feature at $5640 \AA$, which shows lower velocities and a less severe velocity gradient than Si II, more accurately reflects the true photospheric velocity. Hachinger et al. (2006) observed that the velocity determined for $\mathrm{S}_{\text {II }}$ is lower than that determined from Si II $\lambda 6355$ for all SNe types, and that the difference is largest for the HVG SNe. This implies that $\mathrm{Si}$ II $\lambda 6355$ is formed above the photosphere and is not specifically coupled with the photospheric velocity. The velocity evolution of $\mathrm{Si}$ II is dependent on the mass contained in the $\mathrm{Si}$ II line-forming region above the photosphere (deposited by the protrusions of IMEs into the outer layers).

For SN 2004dt, Wang et al. (2006) and Leonard et al. (2005) observed negligible polarization associated with the O I $\lambda 7774$, which Wang et al. interpreted as a spherical distribution of oxygen evenly covering the photodisk. That both Si II and O I occupied the same velocity space, but had very different polarization properties, suggested that portions of the oxygen layer were mixed with IMEs (such as $\mathrm{Si}$ ) with an asymmetric distribution (Höflich et al. 2006). The combination of low polarization for oxygen and the continuum, but with polarized silicon has been observed for other SNe Ia of both LVG and HVG classes such as SNe 2001el, 2006X, and 2009dc (Wang et al. 2003; Patat et al. 2009; Tanaka et al. 2010). The uniformity of oxygen in the outer ejecta of SNe Ia is further demonstrated by Hachinger et al. (2006), who find almost constant line strength for the absorption of O I $\lambda 7774$ for both LVG and HVG SNe at $B$ light curve maximum.

Based on their correlation, Maeda et al. (2010b) suggest that instabilities of both the central and outer layers are caused during the deflagration phase and due to rising plumes. The geometry, and in particular the physics of the runaway and the location of the deflagration to detonation transition, may be affected by other processes such as the progenitor's rotation and its binary companion (Howell et al. 2001; Höflich et al. 2006). In the model of Maeda et al. (2010b), significant heavy elements from the deflagration are mixed into the outer layers, on the side of the ejecta from which an LVG SN Ia is observed.

The polarization of $\mathrm{Si}$ II and the correlation with $\dot{v}_{\mathrm{Si} \text { II }}$ suggest a single offset, with HVG SNe Ia being those with an asymmetric $\mathrm{Si}$ distribution mixed into the outer O layer in the direction of the observer and the products of deflagration offset and receding (positive $v_{\text {neb }}$ ). The Si may be in the form of large clumps or a torus, for example, with higher polarization observed with the line of sight intersecting these structures (with polarization maximized when the principal axis of the asymmetry is oriented at $\sim 30^{\circ}$ from the line of sight; Höflich et al. 2006). At significant angles away from the offset direction, less $\mathrm{Si}$ is found in a thinner layer, more evenly excited by the underlying $\mathrm{Ni}$ substrate, leading to an LVG SN Ia with a lower polarization for Si II $\lambda 6355$ and in which products of deflagration appear to approach the observer (negative $v_{\text {neb }}$ ). These correlations are roughly consistent with the distributions in Figures 1 and 2. The low continuum polarization for both HVG and LVG SNe implies that the products of the detonation, such as ${ }^{56} \mathrm{Ni}$, are spherically distributed in the ejecta. This requires an initial off-center deflagration, followed by an approximately isotropic detonation consistent with the delayed detonation model (Gamezo et al. 2004). An off-center origin of the explosion, in conjunction with the expansion of the ejecta, yields offset (asymmetric) distributions of elements such as $\mathrm{Si}$, with respect to the underlying $\mathrm{Ni}$ (see, e.g., Figure 2 of Höflich et al. 2006), that gives rise to the observed polarization properties of these SNe.

Wang et al. (2003), Wang et al. (2006), and Patat et al. (2009) observed that the Stokes parameters associated with Si II in SNe 2001el, 2004dt, and 2006X formed "loops" on the $Q-U$ plane. These represent the possible difference in axial symmetry between the photosphere and the line-forming region, possibly by clumping or large-scale structure, at different lineof-sight velocities (Kasen et al. 2003).

The two subluminous SNe, 1999by and 2005ke, show low line polarization. Within the framework of single degenerate scenarios, however, such SNe Ia produce ${ }^{56} \mathrm{Ni}$ in the deflagration, whereas during the detonation phase $\mathrm{Si}$-group elements are produced at the expense of ${ }^{56} \mathrm{Ni}$ (Hoflich et al. 1995; Höflich et al. 2002). In models of normal SNe Ia, the majority of the ${ }^{56} \mathrm{Ni}$ is produced during the detonation phase. The fact that line polarization is observed to be strongest in normal SNe Ia and small in subluminous SNe Ia argues against deflagration instabilities as the origin of the observed correlations, especially in LVG SNe Ia (Maeda et al. 2010b).

By bringing together the measurements of $p_{\mathrm{Si}}, \dot{v}_{\mathrm{Si} \text { II }}$, and $v_{\text {neb}}$, a portion of the spectral diversity of normal SNe Ia may be understood in terms of simple orientation effects concerning a single geometry.

The research of J.R.M. is funded through the Sophie \& Tycho Brahe Fellowship. The Dark Cosmology Centre is supported by the DNRF. The research of J.C.W. is supported in part by NSF grant AST-0707769. A.C., J.Q., and P.Z. thank the support of Basal CATA PFB 06/09, FONDAP No. 15010003, and P06045-F (ICM/MIDEPLAN/Chile). P.A.H. was supported by NSF grants 0703902 and 0708855 . The authors are grateful to Stefan Taubenberger for useful discussions concerning the velocity evolution of the faint subclass of SNe Ia.

\section{REFERENCES}

Benetti, S., et al. 2005, ApJ, 623, 1011

Branch, D., Livio, M., Yungelson, L. R., Boffi, F. R., \& Baron, E. 1995, PASP, 107, 1019

Gamezo, V. N., Khokhlov, A. M., \& Oran, E. S. 2004, Phys. Rev. Lett., 92, 211102

Gerardy, C. L., et al. 2007, ApJ, 661, 995

Hachinger, S., Mazzali, P. A., \& Benetti, S. 2006, MNRAS, 370, 299

Höflich, P. 1991, A\&A, 246, 481

Höflich, P. 2006, Nucl. Phys. A, 777, 579

Höflich, P., Gerardy, C. L., Fesen, R. A., \& Sakai, S. 2002, ApJ, 568, 791

Höflich, P., Gerardy, C. L., Marion, H., \& Quimby, R. 2006, New Astron. Rev., 50,470

Hoflich, P., Khokhlov, A. M., \& Wheeler, J. C. 1995, ApJ, 444, 831

Höflich, P., Mueller, E., \& Khokhlov, A. 1993, A\&A, 268, 570

Howell, D. A., Höflich, P., Wang, L., \& Wheeler, J. C. 2001, ApJ, 556, 302

Kasen, D., et al. 2003, ApJ, 593, 788

Khokhlov, A. M. 1991, A\&A, 245, 114

Leloudas, G., et al. 2009, A\&A, 505, 265

Leonard, D. C., Li, W., Filippenko, A. V., Foley, R. J., \& Chornock, R. 2005, ApJ, 632,450

Maeda, K., Taubenberger, S., Sollerman, J., Mazzali, P. A., Leloudas, G., Nomoto, K., \& Motohara, K. 2010a, ApJ, 708, 1703

Maeda, K., et al. 2010b, Nature, 466, 82

Matheson, T., et al. 2008, AJ, 135, 1598

Maund, J., et al. 2010, ApJ, submitted 
Motohara, K., et al. 2006, ApJ, 652, L101

Patat, F., Baade, D., Höflich, P., Maund, J. R., Wang, L., \& Wheeler, J. C. 2009, A\&A, 508, 229

Patat, F., Benetti, S., Cappellaro, E., Danziger, I. J., della Valle, M., Mazzali, P. A., \& Turatto, M. 1996, MNRAS, 278, 111

Perlmutter, S., et al. 1999, ApJ, 517, 565

Phillips, M. M. 1993, ApJ, 413, L105

Phillips, M. M., Wells, L. A., Suntzeff, N. B., Hamuy, M., Leibundgut, B., Kirshner, R. P., \& Foltz, C. B. 1992, AJ, 103, 1632

Riess, A. G., et al. 1998, AJ, 116, 1009

Röpke, F. K., Gieseler, M., Reinecke, M., Travaglio, C., \& Hillebrandt, W. 2006, A\&A, 453, 203

Simon, J. D., et al. 2009, ApJ, 702, 1157
Tanaka, M., et al. 2010, ApJ, 714, 1209

Taubenberger, S., et al. 2008, MNRAS, 385, 75

Turatto, M., Benetti, S., Cappellaro, E., Danziger, I. J., Della Valle, M., Gouiffes, C., Mazzali, P. A., \& Patat, F. 1996, MNRAS, 283, 1

Vinkó, J., Kiss, L. L., Csák, B., Fűrész, G., Szabó, R., Thomson, J. R., \& Mochnacki, S. W. 2001, AJ, 121, 3127

Wang, L., Baade, D., Höflich, P., Wheeler, J. C., Kawabata, K., Khokhlov, A., Nomoto, K., \& Patat, F. 2006, ApJ, 653, 490

Wang, L., Baade, D., \& Patat, F. 2007, Science, 315, 212

Wang, L., \& Wheeler, J. C. 2008, ARA\&A, 46, 433

Wang, L., Wheeler, J. C., \& Höflich, P. 1997, ApJ, 476, L27

Wang, L., et al. 2003, ApJ, 591, 1110

Yamanaka, M., et al. 2009, ApJ, 707, L118 